\title{
Phase II study of concurrent chemoradiotherapy with capecitabine and cisplatin in patients with locally advanced squamous cell carcinoma of the head and neck
}

\author{
JG Kim', SK Sohn', DH Kim', JH Baek', SB Jeon', YS Chae', KB Lee', JS Park ${ }^{*, 2}$, JH Sohn², JC Kim³ and IK Park \\ 'Department of Oncology/Hematology, Kyungpook National University, College of Medicine, Kyungpook National University Hospital, Daegu, Korea; \\ ${ }^{2}$ Department of Otorhinolaryngology, Kyungpook National University, College of Medicine, Kyungpook National University Hospital, Daegu, Korea; \\ ${ }^{3}$ Department of Radiation Oncology, Kyungpook National University, College of Medicine, Kyungpook National University Hospital, Daegu, Korea
}

\begin{abstract}
We aimed to evaluate the efficacy and safety of concurrent chemoradiotherapy with capecitabine and cisplatin in patients with locally advanced squamous cell carcinoma of the head and neck (SCCHN). In total, 37 patients with stage III or IV SCCHN were enrolled on the study. The chemotherapy consisted of two cycles of intravenous cisplatin of $80 \mathrm{mg} \mathrm{m}^{-2}$ on day I and oral capecitabine $825 \mathrm{mg} \mathrm{m}^{-2}$ twice daily from day I to day 14 at 3-week intervals. The radiotherapy (1.8-2.0 Gy I fraction day ${ }^{-1}$ to a total dose of $70-70.2 \mathrm{~Gy}$ ) was delivered to the primary tumour site and neck. The primary tumour sites were as follows: oral cavity ( $n=6)$, oropharynx $(n=11)$, hypopharynx $(n=8)$, larynx $(n=3)$, nasopharynx $(n=6)$, and paranasal sinus $(n=3)$. After the chemoradiotherapy, 29 complete responses (78.4\%) and 6 partial responses (16.2\%) were confirmed. Grade 3 or 4 neutropenia occurred only in two patients, plus grade 3 febrile neutropenia was observed only in one patient. At a median follow-up duration of 19.8 months, the estimated overall survival and progression-free survival rate at 2-year was 76.8 and $57.9 \%$, respectively. Concurrent chemoradiotherapy with capecitabine and cisplatin was found to be well tolerated and effective in patients with locally advanced SCCHN.

British Journal of Cancer (2005) 93, I I I7- I I2I. doi: I0.1038/sj.bjc.6602849 www.bjcancer.com

Published online 25 October 2005

(c) 2005 Cancer Research UK
\end{abstract}

Keywords: capecitabine; chemoradiotherapy; cisplatin; head and neck cancer

The majority of patients with squamous cell carcinoma of the head and neck (SCCHN) present a locoregionally advanced disease, which is associated with a poor prognosis despite treatment with surgical resection or radiation, or both (Vokes et al, 1993). Since the effects of treatment on functional abilities, such as speech and eating, are additional factors to consider in patients with SCCHN, recent attempts to improve the major end points of treatment (local control, organ preservation, and overall survival) have focused on the use of radiotherapy with concurrent chemotherapy (Brizel et al, 1998; Wendt et al, 1998; Calais et al, 1999; Pignon et al, 2000). Generally, cisplatin combined with 5-fluorouracil (5FU) have been considered as one of standard regimen for concurrent chemoradiotherapy (Browman et al, 1994; Brizel et al, 1998; Wendt et al, 1998; Calais et al, 1999). However, the adverse effects of 5-FU, such as oral mucositis, which is an additive complication to radiation, or bone marrow suppression, can result in treatment-related hospitalisation or mortality, thereby compromising the quality of life and compliance to treatment (Wendt et al, 1998).

\footnotetext{
*Correspondence: Dr JS Park, Department of Otorhinolaryngology, Kyungpook National University Hospital, 50 Samduck 2-Ga, Jung-Gu, Daegu 700-7I2, Korea; E-mail: jkk2 Ic@mail.knu.ac.kr

Received 8 August 2005; revised 28 September 2005; accepted 28 September 2005; published online 25 October 2005
}

The oral fluoropyrimidine capecitabine (Xeloda ${ }^{\circledR}$; Hoffmann-La Roche) was rationally designed to preferentially generate $5-\mathrm{FU}$ in tumour tissue and mimic continuous-infusion 5-FU. This tumour selectivity is achieved through exploiting the significantly higher activity of thymidine phosphorylase (TP) in many tumour tissues compared with healthy tissue (Miwa et al, 1998; Schuller et al, 2000). The expression of this enzyme is enhanced in tumour areas with poor perfusion, hypoxia, and acidosis, a situation found in most advanced HNSCC. Moreover, there is evidence that radiation leads to the upregulation of TP expression (Sawada et al, 1999). In a preclinical study, capecitabine given orally resulted in consistently higher tissue-to-plasma 5-FU concentration ratios than 5-FU administered intravenously (Ishikawa et al, 1998). In addition, capecitabine has also exhibited antitumour activity when given as a monotherapy or in combination with cisplatin in patients with various solid tumours as well as in advanced HNSCC (Blum et al, 1999; Van Cutsem et al, 2001; Kim et al, 2002; Pivot et al, 2003; Park et al, 2004).

Furthermore, since the key side effects of capecitabine are hand-foot syndrome and diarrhoea, which overlap little with the side effects of cisplatin or radiation, capecitabine can be a good chemotherapeutic agent in concurrent chemoradiotherapy for SCCHN.

Accordingly, the current phase II study was conducted to evaluate the efficacy and safety of concurrent chemoradiotherapy with capecitabine and cisplatin for locally advanced SCCHN. 


\section{PATIENTS AND METHODS}

\section{Eligibility}

All the patients involved in the current study had measurable, histologically or cytologically confirmed, locoregionally advanced stage III or IV SCCHN arising from the oral cavity, pharynx, larynx, or paranasal sinuses. The patients were 20-75 years of age with a performance status of $0-2$ on the Eastern Cooperative Oncology Group (ECOG) scale. Plus, adequate haematological (WBC count $\geqslant 4 \times 10^{9} 1^{-1}$, platelet count $\geqslant 100 \times 10^{9} 1^{-1}$, haemoglobin $\geqslant 9 \mathrm{~g} \mathrm{dl}^{-1}$ ), renal (serum creatinine $\leqslant 1.5 \mathrm{mg} \mathrm{dl}^{-1}$ and creatinine clearance $\geqslant 50 \mathrm{ml} \mathrm{min}{ }^{-1}$ ), and hepatic (total bilirubin $\leqslant 2.0 \mathrm{mg} \mathrm{dl}^{-1}$ and serum transaminase level $\leqslant 3$ times the upper limit of the normal range) levels were also required. Patients were ineligible if they had previously received chemotherapy or radiation therapy, or had other severe medical illnesses, distant metastasis, another active malignancy in the last 5 years, except treated nonmelanoma skin cancer or cervical dysplasia, or a history of anaphylaxis to drugs. The institutional review board of the authors' institution approved the protocol, and written informed consent was obtained from all patients before enrollment.

\section{Study treatment}

The administration schedule is shown in Figure 1. Capecitabine $825 \mathrm{mg} \mathrm{m}^{-2}$ b.i.d with pyridoxine $100 \mathrm{mg}$ t.i.d was given on days $1-14$, followed by a 7-day rest period. The capecitabine was supplied as film-coated tablets at two dose strengths, 150 and $500 \mathrm{mg}$, while the cisplatin $80 \mathrm{mg} \mathrm{m}^{-2}$ was administered through a 1-h intravenous infusion on the first day of each cycle. Pre- and post intravenous hydration and appropriate antiemetics were also administered to prevent renal toxicity and emesis. Two cycles of chemotherapy were repeated every 3 weeks.

Radiotherapy (1.8-2.0 Gy 1 fraction day ${ }^{-1}$ to a total dose of $70-$ $70.2 \mathrm{~Gy}$ ), administered 5 days per week, was delivered to the primary tumour site and neck, and was targeted to begin on the first day of chemotherapy. Patients underwent a complete dental evaluation and treatment as early as possible before the initiation of radiotherapy. Every effort was made to continue the radiation on schedule.

\section{Dose modification}

The protocol plan was to continue the study treatment despite mucositis or dermatitis. However, if grade 3 or 4 capecitabinerelated haematological or nonhaematological toxicity, such as diarrhoea and hand-foot syndrome, occurred (not including

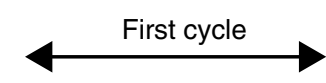

Day $123 \ldots$.

Capecitabine P.O.

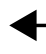

Irradiation: 70 - 70.2 Gy

Figure I Administration schedule of concurrent chemoradiotherapy with capecitabine and cisplatin in patients with squamous cell carcinoma of the head and neck. i.v: intravenous; PO: per oral. radiation-related toxicity), capecitabine was withheld until the toxicity had improved by at least two grade levels. Subsequent capecitabine doses then required a $20 \%$ dose reduction. The dose of cisplatin was reduced to $50 \%$ if the calculated creatinine clearance level was $30-50 \mathrm{ml} \mathrm{min}^{-1}$. No cisplatin was administered if the creatinine clearance level was less than $30 \mathrm{ml} \mathrm{min}^{-1}$. In the presence of myelosuppression (WBC count $<4 \times 10^{9} \mathrm{l}^{-1}$ or platelet count $<100 \times 10^{9} 1^{-1}$ ), a persisting fever that exceeded $38^{\circ} \mathrm{C}$, or other clinically apparent infections, a cycle could be postponed for 1 week or interrupted if this was judged to be necessary in the opinion of the attending physicians.

\section{Surgery}

Salvage surgery of the primary tumour site was recommended for operable patients with a respectable disease, who failed to achieve a complete response (CR) after the end of the chemoradiotherapy. The extent of surgery varied, ranging from laser resection or a wide excisional biopsy to a complete resection of the oral cavity or tonsillar primary. A modified neck dissection could also be performed. The surgery was carried out routinely $6-8$ weeks after the chemoradiotherapy.

\section{Study assessments}

Before being enrolled on the trial, all patients underwent a full medical history and physical examination, blood tests, computed tomography (CT) or magnetic resonance imaging of the head and neck, and chest X-ray (CT of the chest if the patient's low neck nodes were involved). Assessment of the tumour response by clinical examination and CT scanning took place 6 weeks after completing the chemoradiation therapy. A biopsy or fine needle aspiration cytology to determine the pathologic response was not routinely performed. The definitions of CR, partial response (PR), stable disease (SD), and progressive disease (PD) were based on the standard definitions established by the WHO (1979). The patients were monitored for toxicity (medical interview, physical examination, and complete blood count) throughout the treatment. Complete blood counts and chemistry were performed every week until the end of the chemoradiotherapy. Systemic toxicity resulting from treatment was graded according to the National Cancer Institute Common Toxicity Criteria (NCI-CTC) version 3.0. Acute radiation toxicities were graded according to the European Organization Therapy Oncology Group (EORTC-RTOG) toxicity criteria. Hand-foot syndrome was graded 1-3, as defined in previous capecitabine clinical studies (Blum et al, 1999).

\section{Statistical analysis}

As a phase II study, the primary end point was to evaluate the response rate, while toxicity, progression, and overall survival were the secondary end points. For sample size calculation, the current trial used a two-stage optimal design, as proposed by Simon (1989), with a $90 \%$ power to accept the hypothesis and $5 \%$ significance to reject the hypothesis. Plus, the current trial was designed to detect a response rate of $90 \%$ as compared to a minimal, clinically meaningful response rate of $70 \%$. Allowing for a follow-up loss rate of $10 \%$, the total sample size was 36 patients with a measurable disease. Time to progression was measured as the time from the initiation of therapy until death of disease or toxicity, appearance of new lesions, or a greater than $25 \%$ increase of the indicator lesions over the previous smallest size. Overall survival was measured from the initiation of therapy to the date of the last follow-up or any cause of death. Progression-free and overall survival analyses were all estimated using the KaplanMeier method. The statistical data were obtained using an SPSS software package (SPSS 11.0 Inc. Chicago, IL, USA). 


\section{RESULTS}

\section{Patient characteristics}

A total of 37 patients were enrolled in the current study from April 2003 to May 2004 at Kyungpook National University Hospital, Daegu, Korea. The characteristics of the patients are summarised in Table 1. The median age of the patients was 61.0 years (range, $35-75$ years), and $31(83.8 \%)$ patients were male. Most of the patients $(89.2 \%)$ had a good performance status (ECOG 1$)$. The primary sites of the tumours were as follows: oral cavity $(n=6)$, oropharynx $(n=11)$, hypopharynx $(n=8)$, larynx $(n=3)$, nasopharynx $(n=6)$, and paranasal sinus $(n=3)$. A total of 22 patients $(59.5 \%)$ had a stage III disease, while the remaining 15 patients were stage IV. In all, 16 patients $(43.2 \%)$ had an $\mathrm{N} 2(n=15)$ or N3 $(n=1)$ status before treatment.

\section{Response and survival}

Of the 37 patients, $34(91.2 \%)$ completed the planned treatment, with the remaining three being lost to follow-up or patient refusal. All efficacy data are reported using the intent-to-treat patient population. After the chemoradiotherapy, 29 CRs (78.4\%) and 6 PRs $(16.2 \%)$ were confirmed, giving an overall clinical response rate of $94.6 \%$ (95\% CI; $87.0-102.2 \%)$. The primary site CR was $80.0 \%$ (28 out of 35 ) and metastatic lymph node CR was $70.4 \%$ (19 out of 27) (Table 2). Four out of six patients with nasopharyngeal

Table I Patient characteristics

\begin{tabular}{|c|c|}
\hline Characteristic & Number of patients, $\mathbf{N}=\mathbf{3 7}(\%)$ \\
\hline \multicolumn{2}{|l|}{ Age (years) } \\
\hline Median (range) & $61(35-75)$ \\
\hline Male/female & $31(83.8) / 6(16.2)$ \\
\hline \multicolumn{2}{|l|}{ ECOG performance status } \\
\hline I & $33(89.2)$ \\
\hline 2 & $4(10.8)$ \\
\hline \multicolumn{2}{|l|}{ Site of primary tumour } \\
\hline Oral cavity & $6(16.2)$ \\
\hline Oropharynx & II (29.7) \\
\hline Hypopharynx & $8(21.6)$ \\
\hline Larynx & $3(8.1)$ \\
\hline Paranasal sinus & $3(8.1)$ \\
\hline Nasopharynx & $6(16.2)$ \\
\hline \multicolumn{2}{|l|}{ Histologic classification } \\
\hline Well differentiated & $8(21.6)$ \\
\hline Moderately differentiated & $10(27.0)$ \\
\hline Poorly or undifferentiated & II (29.7) \\
\hline Unspecified & $8(21.6)$ \\
\hline \multicolumn{2}{|l|}{ Stage } \\
\hline III & $22(59.5)$ \\
\hline IV & $15(40.5)$ \\
\hline \multicolumn{2}{|l|}{$T$ classification } \\
\hline $\mathrm{TI}$ & $3(8.1)$ \\
\hline $\mathrm{T} 2$ & $15(40.5)$ \\
\hline T3 & $13(35.1)$ \\
\hline T4 & $6(16.2)$ \\
\hline \multicolumn{2}{|l|}{ N classification } \\
\hline NO & $9(24.3)$ \\
\hline $\mathrm{NI}$ & $12(32.4)$ \\
\hline N2 & $15(40.5)$ \\
\hline N3 & I (2.7) \\
\hline
\end{tabular}

tumour showed CR and remaining two patients PR. Among the eight patients who failed to achieve CR after the chemoradiotherapy, three patients underwent surgery and two patients received salvage chemotherapy. At the time of the present evaluation, 13 patients had developed disease progression or recurrence (five primary tumour, three - regional lymph node, two - both primary tumour and regional lymph node, and three - distant metastases to the bone), and seven patients had died of disease progression. The median survival time had not yet been reached at a median followup duration of 19.8 months (range, 3.1-27.5 months), while the estimated overall survival and progression-free survival rate at 2year was $76.8 \pm 8.5$ and $57.9 \pm 11.1 \%$, respectively (Figure 2).

Table 2 Tumour response (intent-to-treat analysis, $N=37$ )

\begin{tabular}{lcccccc}
\hline & \multicolumn{5}{c}{ Response (\%) } \\
\cline { 2 - 7 } Variable & CR & PR & SD & PD & Response rate \\
\hline Primary site & $28 / 35(80.0)$ & $5 / 35(14.3)$ & $2 / 35(5.7)$ & $0 / 35$ & $33 / 35(94.3)$ \\
Lymph nodes & $19 / 27(70.4)$ & $5 / 27(18.5)$ & $2 / 27(7.4)$ & $1 / 27(3.7)$ & $24 / 27(88.9)$ \\
Overall & $29 / 37(78.4)$ & $6 / 37($ ( 6.2$)$ & $1 / 37(2.7)$ & $1 / 37(2.7)$ & $35 / 37(94.6)$ \\
\hline
\end{tabular}

$\mathrm{CR}=$ complete response; $\mathrm{PR}=$ partial response; $\mathrm{SD}=$ stable disease; $\mathrm{PD}=$ progressive disease.
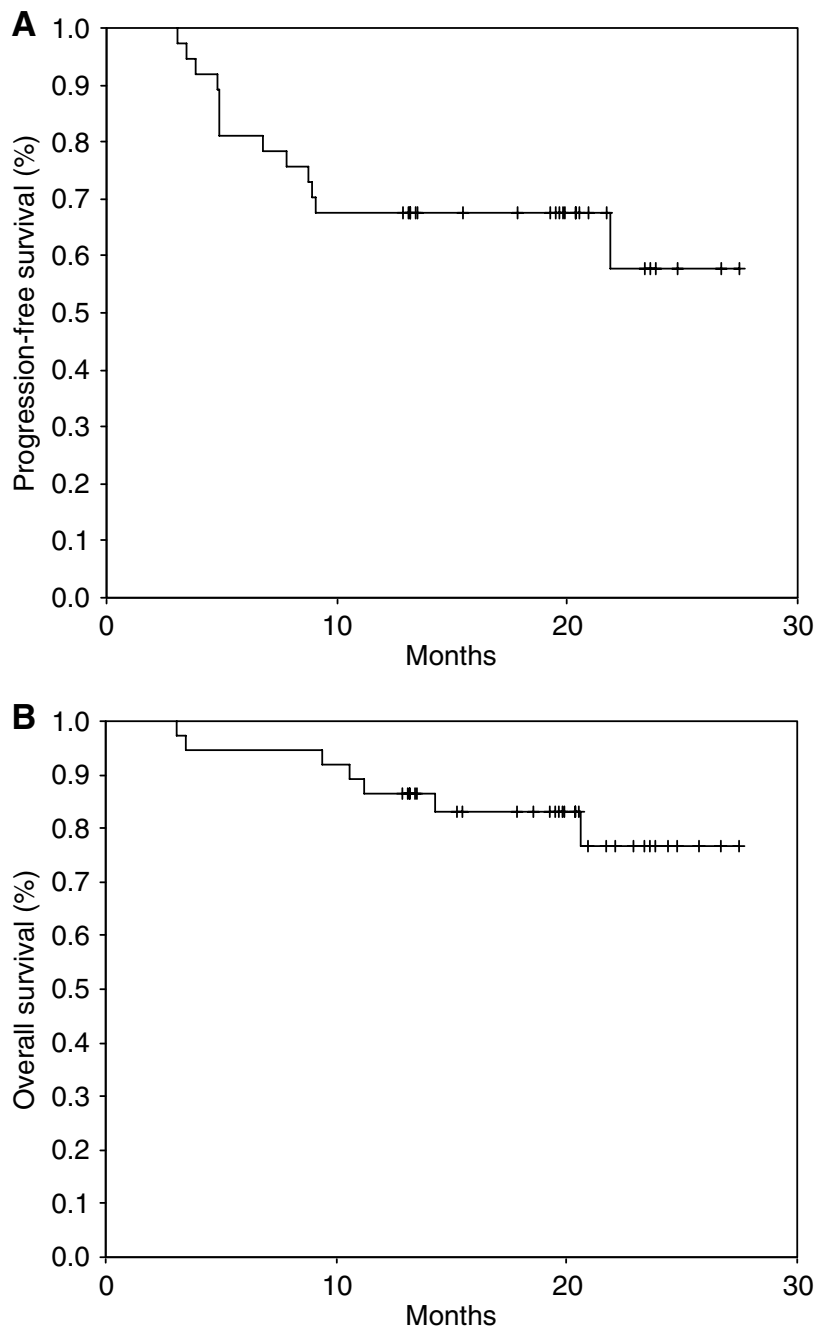

Figure 2 Progression-free survival (A) and overall survival (B) for all patients. 
Table 3 Acute toxic effects $(N=37)$

\begin{tabular}{|c|c|c|c|c|c|}
\hline & \multicolumn{4}{|c|}{ Grade (\% of patients) } & \multirow{2}{*}{$\begin{array}{c}\text { Grade } \\
3 / 4(\%)\end{array}$} \\
\hline & $\mathbf{I}$ & 2 & 3 & 4 & \\
\hline \multicolumn{6}{|l|}{ Haematologic } \\
\hline Anaemia & $13(35.1)$ & $6(16.2)$ & $2(5.4)$ & I (2.7) & 8.1 \\
\hline Leukopenia & $6(16.2)$ & $8(21.6)$ & $2(5.4)$ & I (2.7) & 8.1 \\
\hline Neutropenia & $7(18.9)$ & $6(16.2)$ & I (2.7) & I (2.7) & 5.4 \\
\hline Thrombocytopenia & $12(32.4)$ & $2(5.4)$ & I (2.7) & & 2.7 \\
\hline Febrile neutropenia & & & I (2.7) & & 2.7 \\
\hline \multicolumn{6}{|l|}{ Nonhaematologic } \\
\hline Nausea & || (29.7) & $12(32.4)$ & $5(13.5)$ & I (2.7) & 16.2 \\
\hline Vomiting & $9(24.3)$ & $10(27.0)$ & $3(8.1)$ & & 8.1 \\
\hline Mucositis & $2(5.4)$ & $10(27.0)$ & $17(45.9)$ & $8(21.6)$ & 67.6 \\
\hline Dermatitis (in-field) & $3(8.1)$ & $12(32.4)$ & $7(18.9)$ & $2(5.4)$ & 24.3 \\
\hline Diarrhoea & $4(10.8)$ & $3(8.1)$ & I (2.7) & & \\
\hline Hand-foot syndrome & $12(32.4)$ & $4(10.8)$ & & & \\
\hline
\end{tabular}

Locoregional control rate of the disease at 2-year was $72.6 \pm 7.4 \%$. For the patient group except those with nasopharyngeal tumor, the estimated overall survival and progression-free survival rate at 2 year was $81.3 \pm 9.8$ and $74.2 \pm 7.9 \%$, respectively.

\section{Toxicity}

All 37 patients were assessable for toxicity. The haematologic and nonhaematologic toxicities that occurred during the current study are summarised in Table 3 . The most severe haematologic adverse event was neutropenia, which occurred with a grade $3 / 4$ intensity in two patients (5.4\%). Plus, grade 3 febrile neutropenia was observed in one patient (2.7\%). Although this case was successfully treated with antibiotics and G-CSF, the patient withdrew their consent after this experience. No treatment-related death occurred during this study. Mucositis and dermatitis, as expected from a combination of radiation with an effective chemotherapeuticsensitising agent, were the most common nonhaematological toxicities. Grade 3/4 mucositis and dermatitis was observed in 67.6 and $24.3 \%$, respectively. Grade 2 hand-foot syndrome, a complication of capecitabine, occurred only in four patients $(10.8 \%)$. The dose of capecitabine was reduced in two cycles due to neutropenia or diarrhoea, and cisplatin omitted from one cycle because of nephrotoxicity. The second cycle of chemotherapy was delayed in nine patients for the following reasons: haematological toxicity $(n=7)$, persistent fever $(n=1)$, and patient refusal $(n=1)$. The dose intensity of capecitabine and cisplatin was well maintained throughout the study cycles.

\section{DISCUSSION}

Many studies have demonstrated chemotherapy and radiotherapy to be highly effective in increasing the survival of patients with an unresectable disease. Moreover, concurrent chemoradiotherapy and induction chemotherapy have been established as an appropriate standard of care for patients with locally advanced SCCHN. However, no standard concurrent chemoradiotherapy regimen has been defined.

Therefore, the present phase II study was designed to evaluate the efficacy and toxicity of capecitabine instead of 5-FU, a commonly used agent, in combination with cisplatin for concurrent chemoradiotherapy in patients with locally advanced SCCHN. In the current study, the clinical CR rate $(78.4 \%)$, locoregional control rate $(72.6 \%$ at 2 -year), and progression-free survival rate $(57.9 \%$ at 2 years) following treatment with the present regimen, which can be administered on an outpatient basis, were comparable with previous results reported for 5-FU and platinum-based concurrent chemoradiotherapy, although the follow-up period was relatively short to compare the survival rate directly (Calais et al, 1999; Adelstein et al, 2000, 2003). For example, concurrent chemotherapy with infusion of 5-FU and cisplatin arm achieved a CR rate of $49.4 \%$ and 3-year overall survival rate of $27 \%$ in a randomised study compared with concurrent chemoradiotherapy with radiation therapy alone (Adelstein et al, 2003).

Since the efficacy and favourable safety profile of capecitabine have been clearly demonstrated in recent large phase III studies comparing capecitabine with intravenous 5-FU plus leucovorin for metastatic colorectal cancer (Hoff et al, 2001; Van Cutsem et al, 2001), capecitabine has been widely used in the treatment of breast cancer, stomach cancer, and other solid tumours (Blum et al, 1999; Kim et al, 2002, 2003). Capecitabine also offers a number of potential advantages as a chemoradiosensitiser in concurrent chemoradiotherapy. Daily administration mimicking the continuous infusion of 5-FU can act as a radiosensitiser for every fraction of radiotherapy. Furthermore, its mode of activation by TP and radiotherapy concentrates it within tumour cells, raising the prospect of better tumour control. Given these advantages, several studies have demonstrated that concurrent chemoradiotherapy using capecitabine, with a dose ranging from 800 to $825 \mathrm{mg} \mathrm{m}^{-2}$ b.i.d, in combination with cisplatin or oxaliplatin is effective and has a low toxicity profile in the neoadjuvant setting of rectal cancer or locally advanced oesophageal cancer (Rodel et al, 2003; Hofheinz et al, 2005; Kim JC et al, 2005; Kim SB et al, 2005).

Oral mucositis and myelosuppression are the most serious complications of concurrent chemoradiotherapy for SCCHN, resulting in a reduced compliance to treatment or sometimes mortality. In the present study, mucositis was the most common adverse effect observed, with a grade 3 or 4 intensity in $67.6 \%$ of the patients. In a randomised study by Calais et al (1999), the incidence of grade 3 or 4 mucositis in concurrent chemoradiotherapy with 5 -FU and cisplatin was $71 \%$, which was higher than with radiotherapy only (39\%). The incidence of mucositis was not so different between chemoradiotherapy with capecitabine/ cisplatin and 5-FU/cisplatin. Meanwhile, grade 3 or 4 neutropenia occurred only in two patients (5.4\%), plus grade 3 febrile neutropenia was observed in patient $(2.7 \%)$ in the current study. These incidences of haematologic toxicities were significantly different from previous studies using 5-FU-containing regimens, where the incidence of grade 3 or 4 leukopenia was $29-81 \%$ (Vokes et al, 2000; Adelstein et al, 2003). Recently, taxanes, such as paclitaxel and docetaxel, which exhibit activity against SCCHN, are being increasingly used for concurrent chemoradiotherapy to improve the treatment outcome (Kies et al, 2001; Tishler et al, 2002; Katori et al, 2004). Kies et al (2001) reported that concomitant infusional paclitaxel and fluorouracil, oral hydroxyurea, and radiation therapy achieved a clinical CR rate of $69 \%, 3-$ year locoregional control rate of $86 \%$, and 3-year overall survival rate of $60 \%$ in patients with locally advanced SCCHN. Thus, when considering the high percentage of patients with a T4 and N2/3 disease in this study, the locoregional control rate and overall survival seemed to be promising. However, $34 \%$ of the patients experienced grade 3 or 4 leukopenia, and two patients (3\%) died of treatment-related toxicities. Therefore, taxane-based regimens should be tested in a randomised trial and compared with a less intensive concurrent regimen.

In conclusion, concurrent chemoradiotherapy with capecitabine and cisplatin was found to be well tolerated and effective in patients with locally advanced SCCHN. Accordingly, this regimen can be regarded as an important chemoradiotherapy option for advanced HNSCC, although long-term follow-up is needed to evaluate the late treatment failure and complications. 


\section{REFERENCES}

Adelstein DJ, Lavertu P, Saxton JP, Secic M, Wood BG, Wanamaker JR, Eliachar I, Strome M, Larto MA (2000) Mature results of a phase III randomized trial comparing concurrent chemoradiotherapy with radiation therapy alone in patients with stage III and IV squamous cell carcinoma of the head and neck. Cancer 88: 876-883

Adelstein DJ, Li Y, Adams GL, Wagner Jr H, Kish JA, Ensley JF, Schuller DE, Forastiere AA (2003) An intergroup phase III comparison of standard radiation therapy and two schedules of concurrent chemoradiotherapy in patients with unresectable squamous cell head and neck cancer. J Clin Oncol 21: $92-98$

Blum JL, Jones SE, Buzdar AU, LoRusso PM, Kuter I, Vogel C, Osterwalder B, Burger HU, Brown CS, Griffin T (1999) Multicenter phase II study of capecitabine in paclitaxel-refractory metastatic breast cancer. J Clin Oncol 17: $485-493$

Brizel D, Albers M, Fisher S, Scher RL, Richtsmeier WJ, Hars V, George SL, Huang AT, Prosnitz LR (1998) Hyperfractionated irradiation with or without concurrent chemotherapy for locally advanced head and neck cancer. $N$ Engl J Med 338: 1798-1804

Browman G, Cripps C, Hodson D, Eapen L, Sathya J, Levine MN (1994) Placebo-controlled randomized trial of infusional fluorouracil during standard radiotherapy in locally advanced head and neck cancer. J Clin Oncol 12: $2648-2653$

Calais G, Alfonsi M, Bardet E, Sire C, Germain T, Bergerot P, Rhein B, Tortochaux J, Oudinot P, Bertrand P (1999) Randomized trial of radiation therapy versus concomitant chemotherapy and radiation therapy for advanced stage of oropharynx carcinoma. J Natl Cancer Inst 91: $2081-2086$

Hoff PM, Ansari R, Batist G, Cox J, Kocha W, Kuperminc M, Maroun J, Walde D, Weaver C, Harrison E, Burger HU, Osterwalder B, Wong AO, Wong R (2001) Comparison of oral capecitabine versus intravenous fluorouracil plus leucovorin as first-line treatment in 605 patients with metastatic colorectal cancer: results of a randomized phase III study. J Clin Oncol 19: 2282-2292

Hofheinz RD, von Gerstenberg-Helldorf B, Wenz F, Gnad U, KrausTiefenbacher U, Muldner A, Hehlmann R, Post S, Hochhaus A, Willeke F (2005) Phase I trial of capecitabine and weekly irinotecan in combination with radiotherapy for neoadjuvant therapy of rectal cancer. J Clin Oncol 23: $1350-1357$

Ishikawa T, Utoh M, Sawada N, Nishida M, Fukase Y, Sekiguchi F, Ishitsuka H (1998) Tumor selective delivery of 5-fluorouracil by capecitabine, a new oral fluoropyrimidine carbamate, in human cancer xenografts. Biochem Pharmacol 55: 1091 - 1097

Katori H, Tsukuda M, Mochimatu I, Ishitoya J, Kawai S, Mikami Y, Matsuda H, Tanigaki Y, Horiuchi C, Ikeda Y, Taguchi T, Ono M, Yoshida T, Hirose S, Sakuma Y, Yamamoto K (2004) Phase I trial of concurrent chemoradiotherapy with docetaxel, cisplatin and 5-fluorouracil (TPF) in patients with locally advanced squamous cell carcinoma of the head and neck (SCCHN). Br J Cancer 90: 348-352

Kies MS, Haraf DJ, Rosen F, Stenson K, List M, Brockstein B, Chung T, Mittal BB, Pelzer H, Portugal L, Rademaker A, Weichselbaum R, Vokes EE (2001) Concomitant infusional paclitaxel and flourouracil, oral hydroxyurea, and hyperfractionated radiation for locally advanced squamous head and neck cancer. J Clin Oncol 19: $1961-1969$

Kim JC, Kim TW, Kim JH, Yu CS, Kim HC, Chang HM, Ryu MH, Park JH, Ahn SD, Lee SW, Shin SS, Kim JS, Choi EK (2005) Preoperative concurrent radiotherapy with capecitabine before total mesorectal excision in locally advanced rectal cancer. Int J Radiat Oncol Biol Phys 63: $346-353$

Kim SB, Park SI, Kim JH (2005) A phase II trial of preoperative one cycle of induction chemotherapy [capecitabine (CAP), CDDP] followed by concurrent chemoradiation (CRT) in patients with respectable esophageal cancer. J Clin Oncol 23: 323s

Kim TW, Chang HM, Kang HJ, Lee JR, Ryu MH, Ahn JH, Kim JH, Lee JS, Kang YK (2003) Phase II study of capecitabine plus cisplatin as first-line chemotherapy in advanced biliary cancer. Ann Oncol 14: 1115-1120

Kim TW, Kang YK, Ahn JH, Chang HM, Yook JH, Oh ST, Kim BS, Lee JS (2002) Phase II study of capecitabine plus cisplatin as first-line chemotherapy in advanced gastric cancer. Ann Oncol 3: 1893-1898

Miwa M, Ura M, Nishida M, Sawada N, Ishikawa T, Mori K, Shimma N, Umeda I, Ishitsuka H (1998) Design of a novel oral fluoropyrimidine carbamate, capecitabine, which generate 5-fluorouracil selectively in tumors by enzymes concentrated in human liver and cancer tissue. Eur J Cancer 34: $1274-1281$

Park JH, Kim JH, Ahn SD, Lee SW, Shin SS, Kim JC, Yu CS, Kim HC, Kang YK, Kim TW, Chang HM, Ryu MH, Choi EK (2004) Prospective phase II study of preoperative chemoradiation with capecitabine in locally advanced rectal cancer. Cancer Res Treat 36: 354-359

Pignon JP, Bourhis J, Domenge C, Designe L (2000) Chemotherapy added to locoregional treatment for head and neck squamous-cell carcinoma: three meta-analyses of updated individual data. Lancet 355: 949-955

Pivot X, Chamorey E, Guardiola E, Magne N, Thyss A, Otto J, Giroux B, Mouri Z, Schneider M, Milano G (2003) Phase I and pharmacokinetic study of the association of capecitabine-cisplatin in head and neck cancer patients. Ann Oncol 14: $1578-1586$

Rodel C, Grabenbauer GG, Papadopoulos T, Hohenberger W, Schmoll HJ, Sauer R (2003) Phase I/II trial of capecitabine, oxaliplatin, and radiation for rectal cancer. J Clin Oncol 21: $3098-3104$

Sawada N, Ishikawa T, Sekiguchi F, Tanaka Y, Ishitsuka H (1999) $\mathrm{X}$-Ray irradiation induces thymidine phosphorylase and enhances the efficacy of capecitabine (Xeloda) in human xenografts. Clin Cancer Res 5: $2948-2953$

Schuller J, Cassidy J, Dumont E, Roos B, Durston S, Banken L, Utoh M, Mori K, Weidekamm E, Reigner B (2000) Preferential activation of capecitabine in tumor following oral administration in colorectal cancer patients. Cancer Chemother Pharmacol 34: 293-296

Simon R (1989) Optimal two-stage designs for phase II clinical trials. Controlled Clin Trials 10: $1-10$

Tishler RB, Norris Jr CM, Colevas AD, Lamb CC, Karp D, Busse PM, Nixon A, Frankenthaler R, Lake-Willcutt B, Costello R, Case M, Posner MR (2002) A phase I/II trial of concurrent docetaxel and radiation after induction chemotherapy in patients with poor prognosis squamous cell carcinoma of the head and neck. Cancer 95: 1472-1481

Van Cutsem E, Twelves C, Cassidy J, Allman D, Bajetta E, Boyer M, Bugat R, Findlay M, Frings S, Jahn M, McKendrick J, Osterwalder B, Perez-Manga G, Rosso R, Rougier P, Schmiegel WH, Seitz JF, Thompson P, Vieitez JM, Weitzel C, Harper P (2001) Oral capecitabine compared with intravenous fluorouracil plus leucovorin in patients with metastatic colorectal cancer: results of a randomized phase III study. J Clin Oncol 19: 4097-4106

Vokes EE, Kies MS, Haraf DJ, Stenson K, List M, Humerickhouse R, Dolan ME, Pelzer H, Sulzen L, Witt ME, Hsieh YC, Mittal BB, Weichselbaum RR (2000) Concomitant chemoradiotherapy as primary therapy for locoregionally advanced head and neck cancer. J Clin Oncol 18: $1652-1661$

Vokes EE, Weichselbaum RR, Lippman SM, Hong WK (1993) Head and neck cancer. $N$ Engl J Med 328: $184-194$

Wendt TG, Grabenbauer GG, Rodel CM, Thiel HJ, Aydin H, Rohloff R, Wustrow TP, Iro H, Popella C, Schalhorn A (1998) Simultaneous radiochemotherapy versus radiotherapy alone in advanced head and neck cancer: a randomized multicenter study. J Clin Oncol 16: 1318-1324

World Health Organization (1979) WHO Handbook for Reporting Results of Cancer Treatment. WHO Offset Publication No 48. Geneva, Switzerland: World Health Organization 